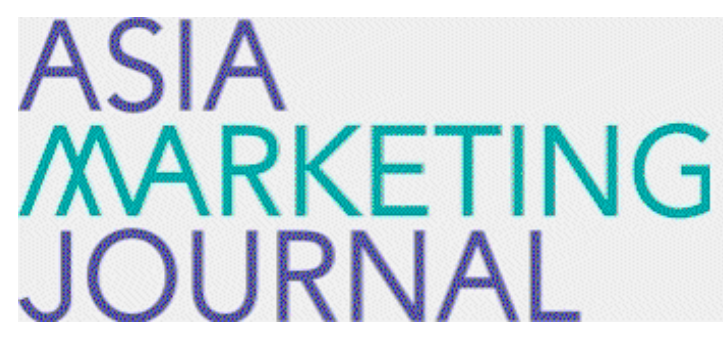

ASIA MARKETING JOURNAL

Volume 17 | Issue 4

Article 2

$1-31-2016$

\title{
Can Threatened Moral Self Make People Prefer Ecological Product?
}

Zhuomin Shi

Wanyi Zheng

Ning Yang

Follow this and additional works at: https://amj.kma.re.kr/journal

Part of the Marketing Commons

\section{Recommended Citation}

Shi, Zhuomin; Zheng, Wanyi; and Yang, Ning (2016) "Can Threatened Moral Self Make People Prefer Ecological Product?," Asia Marketing Journal: Vol. 17 : Iss. 4 , Article 2.

Available at: https://doi.org/10.15830/amj.2016.17.4.21

This Article is brought to you for free and open access by Asia Marketing Journal. It has been accepted for inclusion in Asia Marketing Journal by an authorized editor of Asia Marketing Journal. 


\title{
Can Threatened Moral Self Make People Prefer Ecological Product? \\ - An Eye Tracking Research based on Chinese Face Consciousness*
}

\author{
Zhuomin Shi** \\ Wanyi Zheng*** \\ Ning Yang****
}

Purpose: Social influence has a decisive role in shaping a person's cognition and behavior. Chinese face consciousness, including moral component, is an important part of Chinese traditional culture, which influences people to implement moral behavior. With both eye-tracking technology and traditional questionnaire, this research aims to explore people's moral psychology and the psychological processing mechanisms of Chinese face consciousness, as well as the impact of Chinese face consciousness on the preference for the ecological product.

Method and Data: 75 college and MBA students' eye movement data were collected when they read different kinds of moral materials, as well as data from the subsequent questionnaires. To test the hypothesis, ANOVA analysis and Heat Map analysis were performed. Besides, the PROCESS of bootstrap was used to test mediation effect.

Findings: The results reveal that: 1 . Compared to the moral-situation reading, when subjects read immoral situations, they need more processing time due to the moral dissonance and cognitive load. 2. Compared to the control condition, when threatened moral self is primed, subjects prefer to choose ecological product. 3. Protective face orientation is the mediator between threatened moral self and preference to ecological product.

Key Contributions: First, this study broadens the use of eye-tracking technology in marketing and demonstrates a better understanding of the relationship between morality and consumer behavior in a more scientific way. Second, this study not only distinguishes the meanings between "protective face orientation" and "acquisitive face orientation", but also innovatively validates that when moral self is threatened, consumers tend to choose ecological product as moral compensation in order to protect their face. It can shed light on the promotion of ecological product in practical applications.

Key words: Moral Self, Chinese Face Consciousness, Ecological Product, Eye Tracking.

This work is supported by the National Science Foundation of China (No. 71572205).

** Sun Yat-sen University, China (mnsszm@mail.sysu.edu.cn)

*** Nanfang College of Sun Yat-sen University, China (zwysara@foxmail.com), Corresponding Author

**** Sun Yat-sen University, China 


\section{Introduction}

Social impact has a decisive role in shaping a person's cognition and behavior (Ratner and Kahn 2002). It can mainly be divided into two categories: informational influence and normal influence. Wherein, normal influence is relevant to the individual choice to imitate others in order to obtain approval or to avoid rejection (Zhang and Zhuang 2008). In China, gaining social approval is an obvious expression of gaining face. Chinese people value face and attach great importance to save others' face in social interaction. For example, Chinese parents would avoid criticizing their child in public. Face is an important part of traditional Chinese culture. As Lin (1939) mentioned, face, grace and destiny are the three goddesses which dominate the Chinese society. Face is relatively more powerful than law, and it is the most exquisite standard in social interaction.

As a prevalent concept, face includes two distinctive dimensions in China. Hu (1944) originally proposed that face is divided into "LIAN" and "MIAN". In her definition, LIAN stands for one's integrity and moral reputation, and MIAN is related to public image coming from the visible achievement. With empirical study, Shi et al. not only verified the two dimensions of Chinese face concept, but also explored the connotation of LIAN and MIAN (Shi, Furukawa, and Jin 2011), which are also known as "moral face" and "social face" (King and Myers 1977). As essential component of face, morality is an important part of Chinese culture as well and it has become the inner standards to everyone.

Moral self refers to the moral parts of selfconsciousness, including moral self-evaluation, moral self-image, self-esteem, self-confidence, ideal-self and moral self-control ability (Zhu 1989). As a dimension of moral identity, moral self refers to the self-schemas that serve as mental representations of people's willing to become a moral person and conduct moral behavior (Hardy 2014). When there are conflicts between an individual's behavior and social norms or the individual's internalized moral standards, individual will sense an uncomfortable feeling because of his moral self being threatened. For example, we Chinese people all know we should help the elderly when they fall down, but if people easily get extorted because of offering a hand, they will become more and more apathy. So how will people react to relieve such feelings that moral self is being threatened? Li and $\mathrm{Yu}$ (2013) summarized the coping mechanisms when moral self is threatened, which are cognitive approach, behavioral approach and distance response. Cognitive approach includes moral disengagement and moral justification (Kohlberg 1971). Behavioral approach includes moral behavior (moral compensation and moral licensing) and cleaning behavior (Effron, Miller and Monin 2012). Distance response refers to 
"Pot calling the kettle black" or Double-distancing mechanism (Barkan 2012). Moreover, Conway and Peetz (2012) found that when people recall recent immoral behavior, they tend to choose moral behavior for moral compensation. Thus, when people fail to implement moral behavior, it is easy to prime threatened moral self. Subsequently, in order to relieve cognitive dissonance, people are much more inclined to conduct moral behavior ( $\mathrm{Li}$ and Yu 2013).

To explore the mechanisms behind threatened moral self and moral compensation behavior, we intend to seek support from quantitative research on moral psychology. Notwithstanding, current quantitative research on moral psychology has three following major issues. First, researchers generally use questionnaires or selfreport, which makes the psychological reactions of consumers ignored. However, the psychological reactions of consumers are often the most authentic reflection on the relationship between personal characteristics and behavior (Zahn, et al. 1996). Second, people prefer to use impression management to shape their own image as well as to gain respect from the society. Similarly, they will change attitudes specifically due to "social desirability" (Kwang-kuo 1987). Third, people may not have the ability to report their true thoughts sometimes (Wänke et al. 2002). Due to the reasons above, data using in majority of existing studies are not scientific enough. In order to solve the problem, the researchers start to use psychophysical technologies, such as, heart rate measurement, pupillary response, galvanic skin response, brain waves, eye tracking technology and so on, and they have been applied on different marketing fields such as products, package design, advertising and others, so that research results become more scientific and accurate (Wänke et al. 2002).

Moreover, consumer's decision partly depends on inherent human cognitive processes (Carrington, Neville, and Whitwell 2010). Human sub consciousness is more influential on behaviors than the physical properties (Zaltman 2003). Therefore, using eye tracking technology to explore people's unconscious eye movements becomes more necessary. Meanwhile, consumer's moral choice is significantly influenced by the local culture. Naturally, this study is to investigate the mechanism of consumer's moral behavior in Chinese face culture.

Due to the deteriorating environment and people's growing attention on the environment, more and more academic scholars are exploring the influential factors of moral behavior which can improve environment as well as to promote people to change behavior (Luchs et al. 2010). And this study will focus on how threatened moral self affects Chinese consumers' ecological consumption behavior in Chinese culture.

To sum up, this study tries to make up the literature gap of the research about the ecological behavior and threatened moral self. Specifically, this research aims to inquire the differences of cognitive processing when people face different 
moral conflict situations through eye tracking technology, as well as to explore people's moral psychology and the psychological processing mechanisms under the influence of Chinese face consciousness. It enriches the fields of both face consciousness and moral psychology research. What is more, the conclusions have certain significance to ecological product promotion.

\section{Literature}

\subsection{Morality}

Morality refers to such a situation when people give up personal self-interest, but focus on the needs of others (Kant 2002). Chinese have been taught to behave morally since childhood, because people think moral behavior is commendable and admirable. Therefore, it has already been internalized as important standards in Chinese people's minds. Moral standards are distinct from personal preferences, because moral conviction is generic, which means that moral standards are applicable to anyone (Haidt, Rosenberg, and Hom 2003).

When one realizes the existence of morality, then follows the appearance of moral self. The connotation of moral self is rich. However, there is no universal explanation yet. In this study, we adopt the definition in "Dictionary of Psychology" which is written by Zhu (1989). Moral self re- fers to the moral parts of self-consciousness, including moral self-evaluation, moral self-image, self-esteem, self-confidence, ideal-self and moral self-control ability (Zhu 1989). According to the theory of moral self-management, people seek positive moral balance, and tend to regard themselves as a moral good person (Sherman and Cohen 2006). Nonetheless, since the influential factors of moral behavior are plenty and uncertain, people are unable to do the right things in accordance with social moral standards all the time. As a result, it easily leads to threatened moral self.

Threatened moral self refers to the situation when there are conflicts between personal behavior and social norms or the personal internal moral standards. Namely, moral disorder will result in threatened moral self.

People regard themselves as a moral person because immoral behavior easily leads to selfcensure. Naturally, people tend to consciously change behaviors deviating from moral standards, in order to narrow the gap between moral standards and actual behavior, relieving the feeling coming from threatened moral self (Bandura et al. 1996).

In China, Li and Yu (2013) summarized the coping mechanisms when moral self is threatened and find out three different ways as follows: cognitive approach, behavioral approach and distance response ( $\mathrm{Li}$ and Yu 2013), wherein cognitive approach includes moral disengagement and moral justification (Kohlberg 1971), 
behavioral approach consists of moral behavior (moral compensation and moral licensing) and cleaning behavior (Effron, Miller and Monin 2012) and distance response includes "Pot calling the kettle black" or Double-distancing mechanism (Barkan 2012).

Conway and Peetz (2010) found that when people are asked to recall immoral behavior occurred a long time ago (e.g. more than one year), they appear to be moral consistent. When asked to recall a recent immoral behavior (e.g. last week), they tend to conduct moral compensation and choose pro-social behavior in order to avoid damaging their reputation. Jordan, Mullen, and Murnighan (2011) also found the same result that when moral self is threatened versus not being threatened, people prefer pro-social moral behavior. Therefore, this research will study how threatened moral self influences people's preference to choose ecological products.

\subsection{Ecological Consumption}

Ecological consumption is consumption that will meet consumers' basic and potential needs without damaging the bearing capability of the earth. Furthermore, this kind of consumption behavior can be replicated on a global scale (Hertwich 2005). The product corresponding to ecological consumption is called ecological product. Ecological product has certain moral attributes which reflect the principles of morality, and these attributes may be associated with social issues (e.g. animal protection), environmental issues (e.g. recycling and contamination avoiding) (Luchs et al. 2010), etc. This study develops mainly based on the perspective of pro-social behavior.

Morality is an important self-standard. However, for many consumers, the actual behavior is not always consistent with their moral standards. In order to achieve their internal moral standards, when moral self is threatened, they will prefer conducting moral compensation behavior. Specifically, in this case, people prefer to choose those products with moral attributes so as to regain a positive self-image and the internal standards. That is to say, choosing ecological products may be a good way to relieve the feelings of threated moral self.

Meanwhile, according to Bandura's Social Learning Theory, people's behavior is the result of the interaction combined with internal factors and environmental factors (Myers 2006). Ajzen's Theory of Reasoned Action points out that the intention of behavior depends on attitudes towards the behavior and subjective norm (Wang 2010). Therefore, we have to consider face consciousness as an external factor that affects individual consumption behavior in China. Lu (2005) pointed out that Chinese people are more susceptible to others' opinions and views. They attach more attention to develop a positive moral image in public. In China, doing immoral things means losing face. Shameful one may feel when performing immoral behavior, and 
also it may hurt one's reputation and result in losing face. This study will explore this issue in the context of Chinese face culture.

\subsection{Chinese Face Consciousness}

Lu (2005) pointed out that face is the spirit of China in his paper "Said the 'face" ". He also stated that face seems like a simple word, but still many researchers are confused by it. Face, as an important part of traditional Chinese culture values (Zhou and Belk 2004), has a significant impact on the individual psychological needs and self-realization.

The definitions of face differ from culture to culture. In the west, Chinese face first appeared in "The Chinese Characteristics" which was written by American missionary Arthur $\mathrm{H}$. Smith. In late 19th century, Arthur H. Smith (1984) proposed that "saving face" is the first character of Chinese, pointing out Chinese face contains much more meanings than we can express. Face has already attracted scholars' attention in sociology, anthropology, psychology, and many other research fields. Although there is no universal definition, scholars still endeavor to define it according to their own understandings.

Ho (1976) defined that face is the respect and honor that others acquire in social interaction. From psychological perspective, Chen and Yang (2006) defined face as a psychological construct, and it is part of the individual good im- age shown in public. To sum up, we can see that face is compatible with external social standards, and it means both personal ability and morality that are recognized in society (Jin and Yang 2006).

At first, researchers regard "face" as a whole concept. However, many researchers proved that face has two different dimensions ( $\mathrm{Hu}$ 1944; Brown and Levinson 1978; Chou 1999; Shi, Wu, and Kuang 2014). Shi et al. (2011) also discovered that Chinese consumers' face concern (or face need) is not unidimensional but instead consists of two distinct dimensions, which are "LIAN" and "MIAN". LIAN is linked to one's sense of meeting (or not) internal moral standards, likely to play a relatively small role in shaping a Chinese consumer's desire to buy visibly-consumed luxury products. While MIAN, involving with the gaining and signaling of social success and prestige, is possibly dominant in urging Chinese consumers to purchase luxury products (Shi, Fan, and Ye 2012). Brown and Levinson (1978) proposed that "face" consists of "positive face" and "negative face". The former represents the need of self-worth that is recognized by others, and the latter refers to the needs of adults that are not hampered or forced by others. Chou (1999) proposed "face concern", and categorized as "protective face orientation" and "acquisitive face orientation". In Zhang's study (2012), "face" is divided into "desire to gain face" and "fear of losing face". Scholars divided face into two interrelated but 
different dimensions, and according to the research purpose, this study will use the categories of "protective face orientation" and "acquisitive face orientation" from the perspective of the dynamics of face. "Protective face orientation" means that individuals tend to avoid losing face, and "acquisitive face orientation" means that individuals desire to gain face.

Jap (2010) suggested Chinese face culture influences consumers' values to achieve a positive image or higher social status. Face is the desire of being spoken highly for their social values (Ting-toomey and Kurogi 1998). Hence, when one's moral self is threatened, people are afraid of losing face due to protective face orientation, and as a result, ecological consumption is considered a good way to relieve moral dissonance because pro-social behavior will help people achieve positive reputation and social approval again.

\subsection{Eye Tracking}

Eye tracking technology can automatically record subjects' eye movements when they read text, pictures, video, etc. It sheds light on how people allocate their attentions. Moreover, when using eye tracking technology, the experimental situation which is close to the real world can be easily designed. As a result, research findings can be applied more reliably (Yan and Bai 2004). Nowadays, eye tracking technology has been applied in many fields, such as human-computer interaction research, ergonomics, medical field, advertising and design studies, etc. (Duchowski 2002).

The key of eye movement research is how to filter right eye movement indicators. Through bibliometric analysis of domestic eye movement study from 1980 to 2009, Jiang and Zhang (2011) pointed out, among all the eye movement indicators, fixation duration is the most frequently used, while fixation counts ranks the second. On average, each paper uses three eye movement indicators. Since people acquire information through fixation, fixation is generally utilized to study the cognitive process. Moreover, with the deep study of 251 research papers, the use of eye movement indicators is listed as following in Table 1:

Since the study is to evaluate different information processing, fixation duration, fixation counts and pupil diameter are utilized as judging indicators. Total fixation duration means the sum of fixation time when subjects looking at the target. It also shows the extent of processing difficulty. The more fixation counts indicate the region is more likely to be noticed, while, fixation duration indicates area of interest (Liu, Lai, and Chuang 2011). Rayner's (1998) study on the subjects' reading process found that higher cognitive load increases fixation counts and duration.

Pupil diameter, as a frequently used indicator in many research models, including the learning rate (Nassar 2012), error prediction (Preuschoff, 
〈Table 1〉 Eye movement indicators list used in papers ${ }^{1)}$

\begin{tabular}{|c|c|c|}
\hline Types of indicators & Counts of use (times) & Frequency of use (\%) \\
\hline Fixation duration & 202 & 80.48 \\
\hline Fixation counts & 166 & 66.14 \\
\hline Saccade & 115 & 45.82 \\
\hline regression & 60 & 23.90 \\
\hline Pupil diameter & 52 & 20.71 \\
\hline Fixation frequency & 41 & 16.33 \\
\hline
\end{tabular}

Hart, and Einhäuser 2011), nerves gain (Eldar, Cohen, and Niv 2013), and the decision-making process (Cavanagh et al. 2014), has been confirmed as a reflection on emotion fluctuation (Bradley 2008). Larger pupil diameter means higher cognitive load as well (Yan 2004), namely, the subject needs more effort in cognitive process.

Scholars select eye movement indicators according to the purpose and significance of the study. As this study is related to the individual cognitive processing time, it mainly adopts fixation duration, fixation counts and pupil diameter.

\section{Theoretical Framework and Hypothesis}

Based on the situation of moral psychology research, study on moral psychology and behavior is still in the exploratory stage. Therefore, study with the eye-tracking technology is insufficient. Thus, this study relies on general theory, such as cognitive theory, to put forward the theoretical framework shown in Figure 1. And the discussion next will focus on the interaction of various variables and propose relevant assumptions.

\subsection{Moral Dissonance and Eye Movement Indicators}

Culture is a series of values, beliefs, norms and institutions held by some particular groups (Hofstede 1991), and it influences people's behavior. Eye movement means cognitive process (Underwood and Radach 1998). When facing moral conflict situation, in which personal behavior does not conform to social moral norms, it is easy to produce cognitive dissonance. Festinger (1962) put forward the theory of cognitive dissonance, and its basic principle is people have the tendency to balance. Its core content is that

1) Jiang, Bo and Zhang Jinghua (2011), “A Bibliometric Analysis on Eye Movement Research in China From 1980 to 2009,” Psychological Science, 34(1), 235-239. 


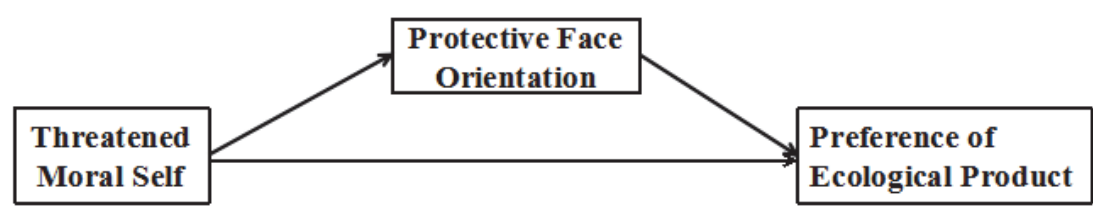

there may be unfitting among different cognitive elements which result in cognitive dissonance. Since people have a tendency to balance, they will spare no effort to reduce the imbalance or disorder pressure, like changing cognition, behavior or obtaining information. This study is mainly focused on moral dimension of cognitive disorders (Lowell 2012).

Morality is an important dimension of selfdefinition. According to the theory of moral self-management, people seek positive moral balance, and tend to think of themselves as a moral and good person (Sherman and Cohen 2006). Moral standards are distinct from personal preferences, because moral conviction is generic, which means that moral standards are applicable to anyone (Haidt, Rosenberg, and Hom 2003). Henderson and Hollingworth (1999) found that when the semantics of an object is inconsistent, it needs longer fixation duration. It also needs more processing time when cognitive effort increases (Tun, Benichov, and Wingfield 2010). Just and Carpenter's (1976) Eye - Brain assumption proposes that, the subject will stare at a certain area as long as he is processing the area. Fixation duration and fixation counts are the indicators to measure cognitive effort.
Therefore, when reading immoral situations, the subjects need longer fixation time and more fixation counts. Thus, hypothesis $1 \mathrm{a}$ and $1 \mathrm{~b}$ are proposed as follows:

H1a: Compared to the moral situations reading, when subjects read immoral situations, they need longer fixation duration.

H1b: Compared to the moral situations reading, when subjects read immoral situations, they need more fixation counts.

With the development of eye tracking technology, the pupil diameter can be automatically recorded. Pupil diameter is susceptible to the autonomic nervous system. In the field of psychology, pupil dilation can be led by fear, cognitive load, emotion fluctuation, suffering, risk, novelty, accidents and conflicts (Laeng, Sirois, and Gredebäck 2012). Critchley et al. (2005) also found that when subjects experience cognitive conflict, they would enlarge the pupil diameter. Thus, hypothesis 1c is proposed as follows:

H1c: Compared to the moral situations reading, when subjects read immoral situations, their pupil diameter will be larger. 


\subsection{Threatened Moral Self and Preference of Ecological Products}

In reality, people emphasize on whether their own behavior meets moral standards. People's global self-integrity depends largely on the extent of perceived self-morality (Dunning 2007), and immoral conduct impairs their self-integrity perception. Hence, when facing immoral behavior, people are more likely to choose moral behavior to regain self-integrity, which is called moral compensation (Sachdeva, Iliev, and Medin 2009). Individuals adopt moral compensation to alleviate the discomfort (Baumeister and Heatherton 1996). In fact, when violating internal moral standards, people may feel uncomfortable because of cognitive dissonance, which requires more cognitive resources. However, people's cognitive resource is finite (Baumeister and Heatherton 1996), so cognitive depletion will lead to moral compensation (Joosten et al. 2014). In Peloza, White and Shang's study (2013), they summarized two reasons why people prefer ecological consumption. One is self-standard and the other is self-discrepancy theory. The implementation of moral conduct is an important self-standard. For some consumers, their cognition is not always transformed into practice, but when individuals failed to implement moral behavior, it would induce guilt. Therefore, in order to eliminate the guilt, people would prefer ecological product (Peloza, White, and Shang 2013). Thus, hypothesis 2 is proposed as follows:
H2: Compared to the control condition, when threatened moral self is primed, people prefer to choose ecological product.

\subsection{The Mediation Effect of Protective Face Orientation}

Culture facilitates the implementation of moral behavior (Peng and Yu 2012). Chinese highly emphasize on personal face. In order to comply with social norms, they select to imitate others. Social influence is mainly divided into two categories: informational influence and normal influence. Informational influence means those effects occurred when one obtaining information through different ways; normal influence refers to the individual choice to imitate others in order to obtain approval or to avoid rejection (Zhang and Zhuang 2008). Zhang and Zhuang (2008) conducted a quantitative study and found that, in the context of companion shopping, normal influence tends to positively affect individual impulsive buying. They also examined that informational influence tends to affect individual impulsive buying through the mediator of protective face orientation (Zhang and Zhuang 2008). Triandis (1989) proposed that behavioral intention is affected by perceived behavioral result, social factors (face, standards, etc.) and affective factors.

In real life, when our social relations are attacked, we will strive to protect face. Cupach and Carson (2012) conducted a study about 
the psychological defense mechanism when face is threatened, and found when personal selfimage is threatened, people will take appropriate psychological defense mechanism to defend. To some extent, it reveals that protective face orientation can be more likely to induce psychological defense mechanism rather acquisitive face orientation. At the same time, when a person does something immoral, it may harm his reputation and face. In order to protect his face, he may choose ecological product to highlight the personal moral character. Thus, hypothesis 3 is proposed as follows:

H3: Protective face orientation is the mediator between threatened moral self and preference for ecological product.

\section{Methodology}

\subsection{Subjects}

83 undergraduate and MBA students were selected randomly, with average age of 24 years old. After omitting the invalid data, 75 subjects remained. All subjects had qualified acuity or their corrected visual acuities are normal, and all subjects had no color blindness, color weakness or other eye diseases.

\subsection{Experiment Instruments}

In our experiment, EYELINK 1000 is used, which is produced by SR Research Enterprise from Canada. The sampling rate is $500 \mathrm{HZ}$; screen resolution is $1280 \times 1024$ pixels; refresh rate is $60 \mathrm{HZ}$. The instrument will automatically record the first fixation location, fixation duration, fixation counts, pupil size and other data.

\subsection{Experiment Design}

The research combines the advanced eye tracking technology with the traditional closeended questionnaire.

Part I : With eye tracking technology, it measures the different cognitive processing which depends on whether the threatened moral self is primed or not. And the experiment scenarios have pretested. After reading the scenarios, one needs to answer questions to confirm the involvement and cognitive processing difficulty (Wan and Rucker 2013).

Part II : Traditional questionnaire. It includes three tasks:

(1) Chinese Face consciousness Scale is referred to the study of Zhang (2012), and the scale has high reliability and good validity.

(2) Interference task.

(3) Measurement of preference to the eco- 
logical product is referred to the study of Shi, Wu, and Kuang (2014).

(4) Mood scale is referred to the study of Mehta, Zhu, and Cheema (2012).

Part III : Fill in personal information, including gender, age and other demographic variables.

\section{Results and Analysis}

\subsection{Manipulation Test on Ecological Product}

Pretest was conducted to manipulate the material of ecological product. Subjects were required to read materials shown in Table 2 , and then answer two questions: "Which automobile brand (A vs. B) is more powerful?" and "Which automobile brand (A vs. B) is more environment-friendly?", followed by a 7-point scale anchored by "Brand A more powerful/ more environment-friendly" (1) and "Brand B more powerful/more environment-friendly" (7). We compared the means to median of 4 with single-sample T-test. The results show automobile brand $\mathrm{A}$ is more powerful than $\mathrm{B}$ $\left(\mathrm{M}_{\text {power level }}=1.81<4, \mathrm{SD}=1.270, \mathrm{p}<0.000\right)$, and automobile brand $\mathrm{B}$ is more environmentfriendly than A ( $\mathrm{M}_{\text {environment-friendly level }}=6.12$ > $4, \mathrm{SD}=1.619, \mathrm{p}<0.000)$. This indicates that the manipulation on ecological products is successful.

\subsection{Analysis of Control Variables}

Through ANOVA analysis, we found that whether to prime threatened moral self or not, there is no significant difference on the mood. Meanwhile, with ANOVA analysis, none of the demographic variables has significant difference on "protective face orientation", "acquisitive face orientation" or preferences of ecological products under a significant level at 0.01 .

〈Table 2〉 Manipulation readings on ecological product

\begin{tabular}{|c|c|c|}
\hline Indicators & Brand A & Brand B \\
\hline Engine Displacement & 1796 & 1798 \\
\hline Maximum Power (KW) & 108 & 73 \\
\hline Maximum Torque (Nm) & 177 & 142 \\
\hline Wheelbase (mm) & 2685 & 2700 \\
\hline Price (10 thousand) & 17 & 21 \\
\hline Comprehensive Fuel Consumption (L/100km) & 7.7 & 4.3 \\
\hline Power Level & $\star \star \star \star \star$ & $\star \star$ \\
\hline Index of Energy Saving & $\star \star$ & $\star \star \star \star \star$ \\
\hline
\end{tabular}




\subsection{Analysis of Eye Movement Indicators}

\subsubsection{Heat Map}

Eye tracking technology can automatically record subject's eye movements and generate some visualization result figures, such as Heat Map, that can be utilized as primary qualitative analysis.

Heat Map is to distinguish the subject's fixation counts on the area according to shade of color. On a specific area, the darker color it shows, the more fixation counts are generated. It indicates this area may catch more attention. On the contrary, lighter color implies fewer fixation counts, suggesting an easily ignored area. In this experiment, Figure 2 and Figure 3 show the heat map of the priming group and the control group respectively. From the following figures we can clearly observe that, compared to the moral-situation reading, when subjects read immoral situations, they produce more fixation counts.

〈Figure 2〉 Immoral Situation

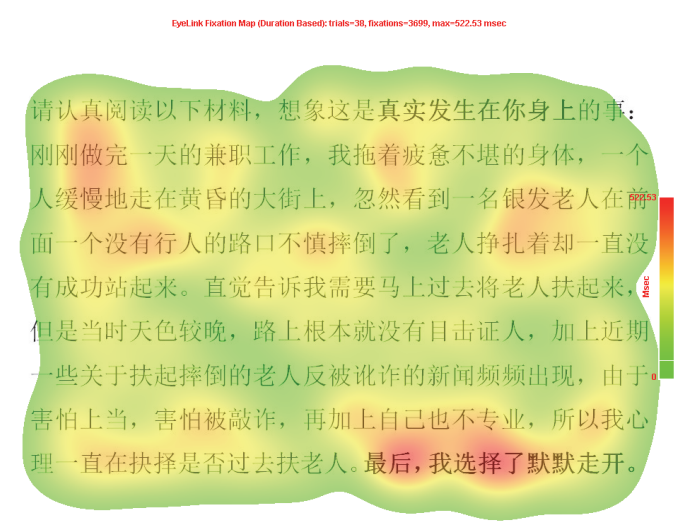

〈Figure 3〉 Moral Situation

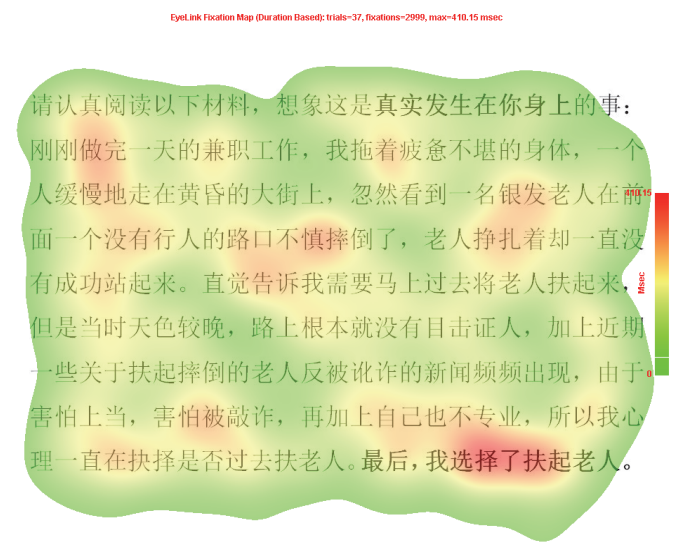

The figures above reveal the qualitative comparison of eye movement data about the subjects. To obtain more accurate and scientific results, SPSS 19.0 will be used to conduct the quantitative analysis.

\subsubsection{Eye Movement Indicators Analysis of Different Moral Situations}

First, with the ANOVA analysis, there is no significant difference on the self-report of the difficulty in cognitive processing between moral and immoral scenarios $(\mathrm{F}(1,73)=0.163, \mathrm{p}=$ $0.688>0.05)$.

Second, through ANOVA analysis, significant difference appears when comparing the fixation duration and fixation counts between immoral group and the control (moral) group (F (1,73) $=5.796, \mathrm{p}=0.019<0.05)$. Compared to the moral situations, when subjects read immoral situations, they need longer fixation duration. Therefore, Hla is supported. At the same time, 
there is marginal significance in the fixation counts $(F(1,73)=4.005, p=0.049<0.05)$. That is to say, when subjects read immoral situations versus moral situations, more fixation counts are generated and thus H1b is supported. Generally, people sometimes may not have the ability to report their true thoughts (Wänke et al., 2002), so the data collected in traditional ways are usually not scientific enough. However, in our findings, no significant difference is shown in pupil diameter, $(\mathrm{F}(1,73)=0.33, \mathrm{p}=0.567$ $>0.05)$. Hence, H1c is not supported. Since plenty of factors could be influential to pupil diameter, such as fatigue, motivation, emotion, attitude of non-verbal communication, cognitive load and so on (Yan, 2004), the measurement of pupil diameter can be easily affected once the experiment did not control other interference variables well.

\subsection{The Effect of Threatened Moral Self on the Preference for Ecological Product}

We tested the difference of ecological product preferences by using ANOVA analysis, and found out when threatened moral self is primed, participants prefer to choose ecological product $(\mathrm{F}(1,34)=4.353, \mathrm{p}=0.045<0.05)$. As a result, $\mathrm{H} 2$ is supported. Although moral schema exists, and everyone can make independent judgments in different moral situations, and ultimately determine their moral behavior, but not everyone can certainly transfer their moral standards into practice. Consequently, when personal behavior does not meet their inner moral standards, threatened moral self appears and urges people to do moral compensation such as pro-social consumption. That is to say, when threatened moral self is primed, people prefer to choose ecological product. Further mediation effect is tested in the following section.

\subsection{Analysis of the Mediation Effect of Protective Face Orientation}

With eye tracking technology, the sample size required is small. If sample size is small, bootstrap method is suitable for analyzing mediation effect (Preacher and Hayes 2004). In this study, we used the PROCESS of bootstrap to test mediation effect, selecting the model $4 \mathrm{oc}^{-}$ curred in the bootstrap processing and bias corrected percentile Bootstrap CI method. Confidence level for confidence interval is $95 \%$ and the bootstrap samples are 5,000.

The test path of mediation effect of "protective face orientation" flows in following way: threatened moral self is primed or not $\rightarrow$ protective face orientation $\rightarrow$ preference of ecological product. The result reveals that the effect of protective face orientation as mediator variable is significant, and interval $(\mathrm{LL95CI}=0.0781$, UL95CI $=$ 1.3838) does not contain 0. Meanwhile, after controlling the variable of protective face orientation, whether to prime threatened moral 
self or not has no effect on the preference of ecological product, and interval (LL95CI = -. 5321, UL95CI $=1.4840$ ) contains 0, which indicates threatened moral self has no effect on the preference for ecological product. As a result, protective face orientation plays a fully mediator. Therefore, H3 is supported. That is to say, protective face orientation is the mediator between threatened moral self and preference for ecological product.

\section{Conclusion and Discussion}

\subsection{Conclusion}

This paper innovatively explores people's different eye movements when facing moral or immoral behavior, and further analyzes the psychological mechanism of the preference for ecological products.

Through empirical research, three essential conclusions can be drawn. First, when subjects read immoral situations compared to moral situations, they need more processing time. That is to say, they need longer fixation time and more fixation counts due to the moral dissonance and cognitive load. Second, compared to the control condition, when threatened moral self is primed, subjects prefer to choose ecological product. Third, protective face orientation is the mediator between threatened moral self and prefer- ence for ecological product. It can shed light on the promotion of ecological product in practical applications.

\subsection{Theoretical Contribution and Practical Implication}

This study has both theoretical and practical contributions.

For theoretical contributions, first of all, this study broadens the use of eye tracking technology in marketing. Through automatically recording eye movement indicators, analysis shows the differences when people read moral or immoral situations. Nowadays, marketing research fields with eye tracking technology include the selection and search behavior, print ads, TV commercials, web advertising, brand design, and so on (Wedel and Pieters 2002), but it remains insufficient. Thus, this study has a breakthrough to introduce eye tracking technology to the field of marketing. Moreover, it demonstrates a better understanding of the relationship between morality and consumer behavior. Besides, the impact of morality on purchase intention and product preferences are complex and worthy of further study.

Secondly, even though the multi-dimensional characteristics of face have been recognized by many scholars, most of the empirical study took face as a whole construct to investigate its impact on consumer behavior. Nevertheless, different dimensions of face may involve different psy- 
chological mechanisms to influence consumer behavior, in which study is relatively scarce (Shi, Wu, and Kuang 2014). Ho (1976) suggested that the gaining or lossing process of face is not an either-or thing. "Desire to gain face" does not mean one will "lose face", since they are not two poles of a one-dimensional variable. Face should be treated as two different social processes. Human beings are always full of contradiction, and they have difficulty making choice. Almost every Chinese desires to gain face and fears of losing face at the same time. Distinction of impact mechanism on consumer behavior further validates that face consciousness is not a single dimension. When moral self is threatened, consumers tend to choose ecological product as moral compensation in order to protect their face.

In addition to the theoretical contributions mentioned above, practical contributions are apparent as well.

First, in the critique of Feuerbach's perceptual human nature, once Marx said, "human beings are not some single and inherent $a b^{-}$ straction, in reality, it is the sum of all social relationship”. American Psychologist Maslow's hierarchy of needs points out that people have needs of emotion, ascription and respect. In China, face culture has deeply affected people's consumption behavior. Furthermore, Chinese put more attention to developing a positive moral image in public. When a person does something immoral, it is very shameful and may affect one's reputation and face. This study explores people's moral psychology and the psychological processing mechanisms of face consciousness. Thus, when moral self is threatened, in order to protect their face, they are more likely to purchase ecological product. Businessmen can also take advantage of this consumer psychology on the ecological product promotion.

Second, the environment problem is not just a threat for the enterprise, and also it may become an opportunity. When it comes to write a text for ads, companies can construct some moral situation reasonably. Currently, ecological products on the market are mixed. Many products are put on green labels to cheat consumers, and it may propel consumers to avoid purchasing ecological products reversely, which is so-called "greenwash". ("Greenwash: used to describe a company, government or organization to declare their certain acts or actions are good for environmental protection, but in fact it is just the opposite." ${ }^{2)}$ ). It is a false environmental advocacy from the essential, as well as a deceptive behavior (Heiskanen 1997). Therefore, to assure the quality of products is the cornerstone to the company.

Third, when consumers lack sufficient knowledge about the ecological product consumption, they may not choose ecological product. So far,

2) The definition of greenwash: http://zh.wikipedia.org/wiki/\%E6\%BC\%82\%E7\%B6\%A0 
many companies pay more attention to nonpollution production process of ecological products, without thinking of the benefits from consumers' point of view. A company should try to reduce consumers' shift cost, otherwise, no buyers are willing to pay more for ecological product. Therefore, marketers should focus on products' benefits for consumers and improve the product delivered value, to urge ecological consumer behavior become a habit. Take a deep consideration of how companies can better fulfill corporate social responsibility and it will be effective for the sustainable development of the company.

\subsection{Research Limitation and Future Research Direction}

This paper explores people's moral psychology and the psychological processing mechanisms of Chinese face consciousness. Also, it provides some inspiration for future research directions. Although some contribution is made in theory and practice, there are also some visible disadvantages.

Firstly, this study is based on college and MBA students, so we should be cautious to generalize the results. The number of sample needs to increase so as to enhance the persuasiveness of the findings. Also, recruiting diverse groups, different in age, gender and so on to increase the representation can also be improvement of this study.

Secondly, before the beginning of the experi- ment, the subjects were informed of their eye movement recording. As a result, a lot of subjects thought that this would affect the results. We cannot completely rule out this possibility, but there is a reason to believe that this is impossible. On one hand, the subjects did not know the purpose of this study and the reasons for using eye-tracking technology. On the other hand, even if the subjects can control eye movements to a certain extent, it will cost them certain self-regulation efforts. That means, the subject is impossible to deliberately manipulate their whole eye movement process.

Thirdly, Bandura's Social Learning Theory proposed human behavior is caused by both internal factors and environmental factors, and the influential factors to the consumer behavior are various and uncertain. This study takes a small perspective which is not fully comprehensive. Thus, more influential factors should be explored in the future.

〈Received November 19. 2015〉

〈Accepted January 20. 2016〉

\section{References}

Bandura, Albert, Claudio Barbaranelli, Gian Vittorio Caprara, and Concetta Pastorelli (1996), "Mechanisms of Moral Disengagement in the Exercise of Moral Agency," Journal of Personality and Social Psychology, 71(2), 
364-374.

Barkan Rachel, Ayal Shahar, Gino Francesca, Ariely Dan (2012), "The Pot Calling the Kettle Black: Distancing Response to Ethical Dissonance," Journal of Experimental Psychology: General, 141(4), 757-773.

Baumeister, Roy and Todd Heatherton (1996), "Self-Regulation Failure: An Overview," Psychological Inquiry, 7(1), 1-15.

Bradley, Margaret M., Laura Miccoli, Miguel A. Escrig, and Peter J. Lang (2008), “The Pupil as a Measure of Emotional Arousal and Autonomic Activation," Psychophysiology, 45(4), 602-607.

Brown, P. and S. C. Levinson (1978), Universals in Language Usage: Politeness Phenomena. England: Cambridge University Press.

Carrington, Michal J., Benjamin A. Neville, and Gregory J. Whitwell (2010), “Why Ethical Consumers Don't Walk Their Talk: Towards a Framework for Understanding the Gap between the Ethical Purchase Intentions and Actual Buying Behaviour of Ethically Minded Consumers," Journal of Business Ethics, 97(1), 139-158

Cavanagh, James F., Thomas V. Wiecki, Angad Kochar, and Michael Frank (2014), "Eye Tracking and Pupillometry Are Indicators of Dissociable Latent Decision Processes," Journal of Experimental Psychology: General, 143(4), 1476-1488.

Chen, Zhizhao and Guoshu Yang (2006), Theoretical Analysis and the Actual Research of
Face Psychology. In Chinese Social Psychological Review (Vol. 2) (pp. 107-160). Beijing: Social Sciences Academic Press.

Chou, Meiling (1999), "Protective and Acquisitive Face Orientation: A Person by Situation Approach to Face Dynamics in Social Interaction," Child Development, 43, 11351160.

Conway, Paul and Johanna Peetz (2012), "When Does Feeling Moral Actually Make You a Better Person? Conceptual Abstraction Moderates whether Past Moral Deeds Motivate Consistency or Compensatory Behavior," Personality and Social Psychology Bulletin, 38(7) : 907-919

Critchley, Hugo D., Joey Tang, Daniel Glaser, Brian Butterworth, and Raymond J. Dolan (2005), "Anterior Cingulate Activity during Error and Autonomic Response," NeuroImage, 27(4), 885-895.

Cupach, William R. and Christine L. Carson (2002), "Characteristics and Consequences of Interpersonal Complaints Associated with Perceived Face Threat," Journal of Social and Personal Relationships, 19(4), 443-462.

Duchowski, Andrew T. (2002), “A BreadthFirst Survey of Eye-Tracking Applications. Behavior Research Methods," Instruments and Computers, 34(4), 455-470.

Dunning, David (2007), "Self-Image Motives and Consumer Behavior: How Sacrosanct Self-Beliefs Sway Preferences in the Marketplace," Journal of Consumer Psychology, 
17(4), 237-249.

Effron Daniel, Miller Dale and Monin Benoît. (2012), "Inventing Racist Roads Not Taken: The Licensing Effect of Immoral Counterfactual Behaviors," Journal of Personality and Social Psychology, 103(6), 916-922.

Eldar, Eran, Jonathan D. Cohen, and Yael Niv (2013), "The Effects of Neural Gain on Attention and Learning," Nature Neuroscience, 16(8), 1146-1153.

Festinger, Leone (1999), Cognitive Dissonance Theory. Zhejiang: Zhejiang People's Publishing House.

Haidt, Jonathan, Evan Rosenberg, and Holly Hom (2003), "Differentiating Diversities: Moral Diversity Is Not like Other Kinds," Journal of Applied Social Psychology, 33 (1), 1-36.

Hardy, Walker, Olsen, Woodbury and Hickman (2014), "Moral Identity as Moral Ideal Self: Links to Adolescent Outcomes," Development Psychology, 50(1), 45-57.

Heiskanen, Eva and Mika Pantzar (1997), "Toward Sustainable Consumption: Two New Perspectives," Journal of consumer policy, 20(4), 409-442.

Henderson, John M. and Andrew Hollingworth (1999), "The Effects of Semantic Consistency on Eye Movements during Complex Scene Viewing," Journal of Experimental Psychology: Human Perception and Performance, 25(1), 210-228.

Hertwich, Edgar G. (2005), "Life Cycle Ap- proaches to Sustainable Consumption: A Critical Review," Environmental Science and Technology, 39(13), 4673-4684.

Ho, David Yau-fai (1976), "On the Concept of Face," American Journal of Sociology, 81 (4), 867-884.

Hofstede, Geert (1991), Cultures and Organizations: Software of the Mind. UK London: McGraw-Hill.

Hu, Hsien Chin (1944), “The Chinese Concept of Face," American Anthropologist, 46(1), 45-64.

Hwang, Kwang-kuo (1987), "Face and Favor: The Chinese Power Game," American Journal of Sociology, 92(4), 944-974.

Jap, Warveni (2010), “Confucius Face Culture on Chinese Consumer Consumption Values toward Global Brands," Journal of International Management Studies, 5(1), 183.

Jiang, Bo and Jinghua Zhang (2011), “A Bibliometric Analysis on Eye Movement Research in China from 1980 to 2009" (in Chinese), Psychological Science, 34 (1), 235-239.

Jin, Yaoji and Guoshu Yang (2006), "Mian”, "Chi" and the Analysis of Chinese People Behavior, In Chinese Social Psychological Review (Vol. 2) (pp. 107-160). Beijing: Social Sciences Academic Press.

Joosten, Anne, Marius Dijke, Alain Hiel, and David Cremer (2014), "Feel Good, Do-Good? On Consistency and Compensation in Moral Self-Regulation," Journal of Business Ethics, 
123(1), 71-84.

Jordan, Jennifer, Elizabeth Mullen, and J. Keith Murnighan (2011), "Striving for the Moral Self: The Effects of Recalling Past Moral Actions on Future Moral Behavior," Personality and Social Psychology Bulletin, 2011, 37(5), 701-713.

Just, Marcel Adam and Patricia A. Carpenter (1976), "Eye Fixations and Cognitive Processes," Cognitive Psychology, 8(4), 441-480.

Kant, Immanuel (2002), Groundwork for the Metaphysics of Morals/ Immanuel Kant, translated by Arnulf Zweig and edited by Thomas E. Hill, Jr. and Arnulf Zweig. Oxford ; New York : Oxford University Press.

King, A. Yeo-ehi and John T. Myers (1977), Shame as an Incomplete Conception of Chinese Culture: A Study of Face. Hong Kong: Chinese University of Hong Kong, Social Research Center.

Kohlberg, Lawrence (1971), From Is to Ought: How to Commit the Naturalistic Fallacy and Get away with It in the Study of Moral Development. In T. Mischel (Ed.), Cognitive Development and Epistemology. New York: Academic Press.

Laeng, Bruno, Sylvain Sirois, and Gustaf Gredebäck (2012), "Pupillometry: A Window to the Preconscious?" Perspectives on Psychological Science, 7(1), 18-27.

Li, Honghan and Yu, Juan (2013), "Moral Self Threats and Corresponding Coping $\mathrm{Me}^{-}$ chanisms" (in Chinese), Journal of Guangxi Normal University : Philosophy and Social Sciences Edition, 49(01), 147-153.

Lin, Yutang (1994), Chinese People. Shanghai: Xuelin Press.

Liu, Han-Chin, Meng-Lung Lai, and HsuehHua Chuang (2011), "Using Eye-Tracking Technology to Investigate the Redundant Effect of Multimedia Web Pages on Viewers' Cognitive Processes," Computers in Human Behavior, 27(6), 2410-2417.

Lowell, Jonathan (2012), "Managers and Moral Dissonance: Self Justification as a Big Threat to Ethical Management," Journal of Business Ethics, 105(1), 17-25.

Lu, Taihong (2005), Chinese Consumer Behavior Report. Beijing: China Social Sciences Press.

Lu, Xun (2005), Said the 'Face'. Beijing: China Renmin University Press.

Luchs, Michael G., Rebecca Walker Naylor, Julie R. Irwin, and Rajagopal Raghunathan (2010), "The Sustainability Liability: Potential Negative Effects of Ethicality on Product Preference," Journal of marketing, 74(5), 18-31.

Mehta, Ravi, Zhu Rui (Juliet), and Amar Cheema (2012), "Is Noise Always Bad? Exploring the Effects of Ambient Noise on Creative Cognition," Journal of Consumer Research, 39(4), 784-799

Myers, David (2006), Social Psychology. Beijing: Posts and Telecom Press. 
Nassar, Matthew R., Katherine M. Rumsey, Robert C. Wilson, Kinjan Parikh, Benjamin Heasly, and Joshua I. Gold (2012), "Rational Regulation of Learning Dynamics by Pupil-Linked Arousal Systems," Nature Neuroscience, 15(7), 1040-1046.

Peloza, John, Katherine White, and Shang, Jingzhi (2013), "Good and Guilt-Free: The Role of Self-Accountability in Influencing Preferences for Products with Ethical Attributes," Journal of marketing, 77(1), 104119.

Peng, Kaiping and Yu, Feng. (2012), "The Psychophysics of Morality: Phenomena, Mechanism and Meaning" (in Chinese), Social Sciences in China (12), 28-45.

Preacher, Kristopher J. and Andrew F. Hayes (2004), "SPSS and SAS Procedures for Estimating Indirect Effects in Simple Mediation Models," Behavior Research Methods, Instruments and Computers, 36(4), 717731.

Preuschoff, Kerstin, Bernard Marius't Hart, and Wolfgang Einhäuser (2011), "Pupil Dilation Signals Surprise: Evidence for Noradrenaline's Role in Decision Making," Frontiers In Neuroscience, 5, 115.

Ratner, Rebecca K. and Barbara E. Kahn (2002), "The Impact of Private versus Public Consumption on Variety-Seeking Behavior," Journal of Consumer Research, 29(2), 246257.

Rayner, Keith (1998), "Eye Movements in Rea- ding and Information Processing: 20 Years of Research," Psychological Bulletin, 124 (3), 372-422.

Sachdeva, Sonya, Rumen Iliev, and Douglas L. Medin (2009), "Sinning Saints and Saintly Sinners: the Paradox of Moral Self-Regulation," Psychological Science, 20(4), 523-528.

Sherman, David K. and Geoffrey L. Cohen (2006), The Psychology of Self-Defense: SelfAffirmation Theory, In (Vol. 118, pp. 1103): Media Source.

Shi, Zhuomin, Lijie Fan, and Jinfeng Ye (2012), "An Empirical Study on Chinese Face and Its Effect on Consumer's Attitude toward Advertising of Luxury" (in Chinese), Nankai Business Review, 15(1), 151-160.

Shi, Zhuomin, Lufang Wu, and Zaoying Kuang (2014), "How Face Consciousness Reverse Pro-Self Behavior? A Study on Sustainable Consumption from the Perspective of Social Value Orientation" (in Chinese), Journal of Marketing Science, 10(2), 59-81.

Shi, Zhuomin, Ichiro Furukawa, and ChunJi Jin (2011), "Cognitive Discrepancy in Chinese "Face": Mian and Lian, and Their Impact on Cognition of Country-of-Origin Image," Frontiers of Business Research in China. 5(2), 163-178.

Smith, Arthur H. (1984), The Chinese Characteristics. New York: FH Revell Co.

Ting-toomey, Stella and Atsuko Kurogi (1998), "Facework Competence in Intercultural Conflict: An Updated Face-Negotiation 
Theory," International Journal of Intercultural Relations, 22(2), 187-225.

Triandis, Harry C. (1989), "The Self and Social Behavior in Differing Cultural Contexts," Psychological Review, 96(3), 506-520.

Tun, Patricia A., Jonathan Benichov, and Arthur Wingfield (2010), "Response latencies in Auditory Sentence Comprehension: Effects of Linguistic versus Perceptual Challenge," Psychology and Aging, 25(3), 730-735.

Underwood, Geoffrey and Ralph Radach (1998), "Eye Guidance and Visual Information Processing: Reading, Visual Search, Picture Perception and Driving," Eye guidance in reading and scene perception, 1-28.

Wan, Echo Wen and Derek D. Rucker (2013), "Confidence and Construal Framing: When Confidence Increases versus Decreases Information Processing," Journal of Consumer Research, 39(5), 977-992.

Wänke, Michaela, Henning Plessner, Tatjana Gärtner, and Wade Malte Friese (2002), "Measuring Implicit Consumer Attitudes and Predicting Brand Choice," Advances in Consumer Research, 29(1), 222.

Wang, Jianming (2010), Resource Conservation and Environmental Protection Behavior and Its Effect Mechanism. Beijing: China Social Sciences Press.

Wedel, Michel and Rik Pieters (2002), "A Review of Eye-Tracking Research in Marketing," Review of Marketing Research, 4(March), 123-147.
Yan, Guoli (2004), The Application of Eye Movement Analysis Method in Psychological Research, Tianjin: Tianjin Education Press. Yan, Guoli and Xuejun Bai (2004), "Eye Movement Analyses in Advertisement Psychology and Its Research Trend" (in Chinese), Psychological Science, 27(2), 459-461.

Zahn, Theodore P., Markus J. P. Kruesi, Susan E. Swedo, Henrietta L. Leonard, and Judith L. Rapoport (1996), "Autonomic Activity in Relation to Cerebrospinal Fluid Neurochemistry in Obsessive and Disruptive Children and Adolescents," Psychophysiology, 33(6), 731-739.

Zaltman, Gerald (2003), How Customers Think: Essential Insights into the Mind of the Market. America: Harvard Business School Press.

Zhang, Xin'an (2012), "Consciousness of Social Face and Conspicuous Consumption of Luxury Products in the Chinese Society" (in Chinese), Journal of Marketing Science, 8(1), 76-94. Zhang, Zhenglin and Zhuang, Guijun (2008), "A Study of Consumer Impulsive Buying Based on the View of Social Influence and Mianzi” (in Chinese), Journal of Management Sciences, 21(6), 66-72.

Zhou, Nan and Russell W. Belk (2004), "Chinese Consumer Readings of Global and Local Advertising Appeals," Journal of Advertising; 33(3), 63-76.

Zhu, Zhixian (1989), Dictionary of Psychology. Beijing: Beijing Normal University Press. 